\title{
Scattering of massless Dirac particles by oscillating barriers in one dimension
}

\author{
C. González-Santander and F Domínguez-Adame \\ GISC. Departamento de Física de Materiales, Universidad Complutense, E-28040 \\ Madrid, Spain \\ C. H. Fuentevilla and E. Diez \\ Laboratorio de Bajas Temperaturas, Universidad de Salamanca, E-37008 Salamanca, \\ Spain
}

\begin{abstract}
We study the scattering of massless Dirac particles by oscillating barriers in one dimension. Using the Floquet theory, we find the exact scattering amplitudes for time-harmonic barriers of arbitrary shape. In all cases the scattering amplitudes are found to be independent of the energy of the incoming particle and the transmission coefficient is unity. This is a manifestation of the Klein tunneling in time-harmonic potentials. Remarkably, the transmission amplitudes for arbitrary sharply-peaked potentials also become independent of the driving frequency. Conditions for which barriers of finite width can be replaced by sharply-peaked potentials are discussed.
\end{abstract}

Keywords: Dirac particles, time-dependent scattering, Klein tunneling PACS: 72.10.-d, 73.23.Hk, 02.30.Jr

\section{Introduction}

Shortly after Dirac formulated his celebrated equation for relativistic electrons $[1,2]$, Klein discovered that Dirac particles undergo anomalous tunneling at high potential barriers [3] (see Ref. [4] for a review). The classical example used to discuss the Klein tunneling is the potential step. When the potential exceeds the rest mass energy, low-energy electrons falling onto the potential step are always transmitted. As a consequence, a strong electrostatic barrier is not able to confine electrons to one side of it [5]. As pointed 
out by Sauter, this effect is quite independent of the potential profile and eventually depends only on its strength [6]. However, due to the unrealizable high potentials required, Klein tunneling was regarded as a curiosity in the context of relativistic quantum mechanics.

With the development of experimental methods to isolate graphene [7] came a renewed interest in Klein tunneling [8, 9, 10]. The reason for this interest is that electrons close to the Fermi energy can be described by the Dirac Hamiltonian for massless particles [11]. In this material Klein tunneling manifests itself as the occurrence of perfect transparency of barriers at normal incidence, as predicted by Katsnelson et al. [12, 13] and later observed in experiments $[14,15]$.

In this work we consider a massless Dirac particle moving in $1+1$ dimensions scattered by a time-dependent potential barrier. Using the Floquet theory we find the exact transmission amplitudes when the barrier is an arbitrary sharply-peaked function at the origin $x=0$, approaching the $\delta$-function limit. We also study the transmission properties of massless Dirac electrons impinging on time-harmonic barriers of finite width and arbitrary shape, and the exact scattering amplitudes will be compared to those corresponding to a sharply-peaked function. The comparison will allow us to establish the conditions under which a finite-width barrier can be replaced by its $\delta$-function limit. We conclude that the $\delta$-function potential is a good approximation to more complex time-dependent barriers whose width is smaller than any other relevant length scale of the problem.

\section{Time-harmonic sharply peaked barrier}

In this section we study the scattering of massless Dirac particles by a time-dependent potential barrier of the form $V(x, t)=g(t) F(x)$. The spatial part of the potential $F(x)$ is assumed to be sharply peaked at the origin $x=0$, approaching the $\delta$-function limit. Importantly, care must be taken when dealing with $\delta$-function potentials in the Dirac equation $[5,16,17]$. The resulting equation is ambiguous if one takes the limit $F(x) \rightarrow \delta(x)$ from the outset. The origin of the ambiguity is the following. Since the Dirac equation is linear in momentum, the wave function itself must be discontinuous at $x=0$ to account for the singular $\delta$-function potential. However, the product of a discontinuous function and the $\delta$-function is mathematically ill defined. This ambiguity can be overcome by solving the corresponding Dirac equation 
for any arbitrary sharply peaked function and then taking the $\delta$-function limit with the constraint $\int_{0^{-}}^{0^{+}} F(x) \mathrm{d} x=1[5]$.

To obtain the proper boundary condition at the origin, we start with the $1+1$ massless Dirac equation

$$
i \dot{\boldsymbol{\psi}}(x, t)=\left[-i \sigma_{x} \frac{\partial}{\partial x}+V(x, t)\right] \boldsymbol{\psi}(x, t),
$$

with $V(x, t)=g(t) F(x)$. The dot indicates the derivative with respect to time and the Pauli matrix $\sigma_{x}$ acts on the two-component wave function $\boldsymbol{\psi}(x, t)$. We take units where the speed of light and $\hbar$ are equal to unity. The appropriate boundary condition for a time-independent sharply-peaked potential was obtained by McKellar and Stephenson in Ref. [5] We now generalize this approach for the time-dependent potential at hand. To this end we cast (1) in the form

$$
\frac{\partial}{\partial x} \boldsymbol{\psi}(x, t)=\widehat{G}(x, t) \boldsymbol{\psi}(x, t)
$$

where

$$
\widehat{G}(x, t)=-\sigma_{x}\left[\frac{\partial}{\partial t}+i g(t) F(x)\right] .
$$

Equation (2a) is solved by a Neumann solution

$$
\boldsymbol{\psi}(x, t)=\widehat{P} \exp \left[\int_{x_{0}}^{x} d x^{\prime} \widehat{G}\left(x^{\prime}, t\right)\right] \boldsymbol{\psi}\left(x_{0}, t\right)
$$

where $\widehat{P}$ is the spatial ordering operator. Taking the limits $x \rightarrow 0^{+}$and $x_{0} \rightarrow 0^{-}$, only the second term of the operator $\widehat{G}$, namely $-i \sigma_{x} g(t) F(x)$, contributes to the integral. This dominant term in the exponential commutes at spatially separated points, so we may set $\widehat{P}=1$. Recalling the constraint $\int_{0^{-}}^{0^{+}} F(x) \mathrm{d} x=1$ we finally arrive at the following boundary condition

$$
\boldsymbol{\psi}\left(0^{-}, t\right)=\exp \left[i g(t) \sigma_{x}\right] \boldsymbol{\psi}\left(0^{+}, t\right)
$$

In the absence of the potential term $(V(x, t)=0)$, solutions of the massless Dirac equation (1) can be written as

$$
\boldsymbol{\psi}_{ \pm}(x, t)=\boldsymbol{\phi}_{ \pm} e^{i E( \pm x-t)}, \quad \boldsymbol{\phi}_{ \pm}=\frac{1}{\sqrt{2}}\left(\begin{array}{c}
1 \\
\pm 1
\end{array}\right)
$$


Notice that $\boldsymbol{\phi}_{ \pm}$are eigenvectors of $\sigma_{x}$ since $\sigma_{x} \boldsymbol{\phi}_{ \pm}= \pm \boldsymbol{\phi}_{ \pm}$. Free solutions are then found to be plane waves travelling to the left or to the right, as deduced from the corresponding current density $j_{ \pm}=\boldsymbol{\psi}_{ \pm}^{\dagger} \sigma_{x} \boldsymbol{\psi}_{ \pm}= \pm 1$.

We now turn to our main goal, the study of the effects of the oscillating barrier on the particle tunneling. The time dependence of the potential will be taken as $g(t)=g_{0}+g_{1} \cos \omega t$ hereafter. As in the case of the Schrödinger equation for an oscillating $\delta$-function potential [18], the Floquet theory allows us to write the wave function in terms of the free solutions (5) as follows

$$
\boldsymbol{\psi}(x, t)=\sum_{n=-\infty}^{\infty} \boldsymbol{A}_{n}(x) e^{-i E_{n} t}, \quad x \neq 0
$$

where $E_{n}=E+n \omega, E$ is a quasi-energy and $n$ is the sideband channel index. Since we are interested in electron transmission across the barrier, we take the following ansatz for the spinors $\boldsymbol{A}_{n}(x)$ in the expansion (6a)

$$
\boldsymbol{A}_{n}(x)=i^{n} \times \begin{cases}\delta_{n 0} e^{i E_{n} x} \boldsymbol{\phi}_{+}+r_{n} e^{-i E_{n} x} \boldsymbol{\phi}_{-}, & x<0, \\ t_{n} e^{i E_{n} x-i g_{0}} \boldsymbol{\phi}_{+}, & x>0 .\end{cases}
$$

The first phase factor $i^{n}$ is introduced for later convenience. Furthermore, the second phase factor $\exp \left(-i g_{0}\right)$ will cancel the term $\exp \left(i \sigma_{x} g_{0}\right)$ after applying the boundary condition (4) since $\exp \left(i \sigma_{x} g_{0}\right) \boldsymbol{\phi}_{+}=\exp \left(i g_{0}\right) \boldsymbol{\phi}_{+}$. These two phase factor have no effect on the transmission probabilities $\left|t_{n}\right|^{2}$ but will simplify the final expression of the transmission amplitudes.

It is straightforward to calculate the time-averaged current density of the wave function (6)

$$
\langle j\rangle=\frac{\omega}{2 \pi} \int_{0}^{2 \pi / \omega} \boldsymbol{\psi}(x, t)^{\dagger} \sigma_{x} \psi(x, t) \mathrm{d} t= \begin{cases}1-R, & x<0, \\ T, & x>0 .\end{cases}
$$

where

$$
R=\sum_{n=-\infty}^{\infty}\left|r_{n}\right|^{2}, \quad T=\sum_{n=-\infty}^{\infty}\left|t_{n}\right|^{2},
$$

are the reflection and transmission probabilities, respectively.

Inserting the ansatz (6) in Eq. (4), multiplying by $\exp \left(i E_{m} t\right), m$ being an arbitrary integer, and time-averaging over one period we get

$$
\delta_{m 0} \boldsymbol{\phi}_{+}+r_{m} \boldsymbol{\phi}_{-}=\sum_{n=-\infty}^{\infty} J_{m-n}\left(g_{1}\right) t_{n} \boldsymbol{\phi}_{+}, \quad m=0, \pm 1, \cdots
$$


where $J_{\ell}(z)$ denotes the Bessel function of the first kind. After multiplying from the left by $\phi_{-}^{\top}$ we conclude that $r_{m}=0$. Consequently, the reflection probability $R$ vanishes. From (8) with $r_{m}=0$, and recalling the orthonormality condition of Bessel functions, one finally gets

$$
t_{n}=J_{-n}\left(g_{1}\right), \quad n=0, \pm 1, \cdots
$$

which satisfies $T=\sum_{n=-\infty}^{\infty}\left|t_{n}\right|^{2}=1$, as expected from the previous result $R=0$.

Several important conclusions can be drawn from the above results. First, the transmission probability is always unity, indicating that Klein tunneling persists even if the barrier is harmonically modulated in time $\left(g_{1} \neq 0\right)$. Nevertheless, the transmission probability through the elastic channel $T_{0}=$ $\left|t_{0}\right|^{2}=J_{0}^{2}\left(g_{1}\right)<1$ is reduced as compared to the static barrier, for which $T_{0}=1$. Second, the transmission amplitudes given by (9) are independent of the incoming energy $E$ and the driving frequency $\omega$. We will see in the next section that the latter is a consequence of the peculiarities of having an infinitely narrow barrier.

\section{Time-harmonic square barrier}

We now consider the scattering from a square barrier of finite width $a$. In this situation, the potential appearing in the massless Dirac equation (1) is

$$
V(x, t)= \begin{cases}V_{0}+V_{1} \cos \omega t, & -a / 2<x<a / 2 \\ 0, & \text { otherwise }\end{cases}
$$

Solutions of the Dirac equation (1) with the time-harmonic potential (10) can be again written down as in Eq. (6a), now including the origin of coordinates $x=0$ since the potential is nonsingular everywhere. Outside the barrier region $(|x|>a / 2)$ the spinors $\boldsymbol{A}_{n}(x)$ are expressed as combination of travelling waves

$$
\boldsymbol{A}_{n}(x)=i^{n} \times \begin{cases}\delta_{n 0} e^{i\left(E_{n} x+V_{0} a / 2\right)} \phi_{+}+r_{n} e^{-i\left(E_{n} x+V_{0} a / 2\right)} \phi_{-}, & x<-a / 2 \\ t_{n} e^{i\left(E_{n} x-V_{0} a / 2\right)} \phi_{+}, & x>a / 2 .\end{cases}
$$

where the phase factors $i^{n}$ and $\exp \left( \pm i V_{0} a / 2\right)$ are introduced for later convenience. Inside the barrier region, solutions are of the form (see, e.g., 
Refs. $[19,20,21])$

$$
\boldsymbol{A}_{n}(x)=\sum_{p=-\infty}^{\infty}\left[A_{p} e^{i\left(E_{p}-V_{0}\right) x} \boldsymbol{\phi}_{+}+B_{p} e^{-i\left(E_{p}-V_{0}\right) x} \boldsymbol{\phi}_{-}\right] J_{n-p}\left(V_{1} / \omega\right),
$$

where $x<|a / 2|$.

Since the potential is nonsingular, the wave function $\boldsymbol{\psi}_{ \pm}(x, t)$ is continuous at $x= \pm a / 2$ and we are led to two equations which we do not write down for brevity. Multiplying them by $\exp \left(i E_{m} t\right), m$ being an arbitrary integer, and time-averaging over one period we get

$$
\begin{aligned}
\delta_{m 0} & =\sum_{p=-\infty}^{\infty} A_{p} e^{i(m-p) \omega a / 2} J_{m-p}\left(V_{1} / \omega\right), \\
t_{m} & =i^{-m} \sum_{p=-\infty}^{\infty} A_{p} e^{-i(m-p) \omega a / 2} J_{m-p}\left(V_{1} / \omega\right), \\
r_{m} & =i^{-m} \sum_{p=-\infty}^{\infty} B_{p} e^{-i(m-p) \omega a / 2} J_{m-p}\left(V_{1} / \omega\right), \\
0 & =\sum_{p=-\infty}^{\infty} B_{p} e^{i(m-p) \omega a / 2} J_{m-p}\left(V_{1} / \omega\right),
\end{aligned}
$$

Using the orthonormality properties of the Bessel functions it is not difficult to show from (12d) that $B_{p}=0$. This implies that backscattering at the discontinuity $x=a / 2$ is suppressed. From (12c) we conclude that $r_{m}=0$ and the reflection coefficient vanishes (backscattering at $x=-a / 2$ is also suppressed). Taking into account the orthonormality properties of the Bessel functions again, one gets $A_{p}=\exp (i p \omega a / 2) J_{-p}\left(V_{1} / \omega\right)$ from (12a). Therefore

$$
\begin{aligned}
t_{m} & =i^{-m} \sum_{p=-\infty}^{\infty} e^{-i(m-p) \omega a} J_{m-p}\left(V_{1} / \omega\right) J_{-p}\left(V_{1} / \omega\right) \\
& =J_{-m}\left(2 \frac{V_{1}}{\omega}\left|\sin \left(\frac{\omega a}{2}\right)\right|\right),
\end{aligned}
$$

where $m=0, \pm 1, \ldots$ and the sum is calculated in the Appendix. In accordance with the previous result $R=0$ for the square barrier, the transmission probability (7) becomes unity in this case. Similarly to what we found for the 
sharply-peaked barrier in (9), the transmission amplitudes are independent of the incoming energy. However, in this case they depend on the driving frequency.

The comparison of the result (13) with the transmission amplitudes for the sharply-peaked barrier given in (9) allows as to define an effective harmonic coupling for the square barrier

$$
g_{1, \mathrm{eff}}(\omega a)=2 \frac{g_{1}}{\omega a}\left|\sin \left(\frac{\omega a}{2}\right)\right|
$$

where now $g_{1}=V_{1} a$. Therefore, we come the important conclusion that the scattering properties of harmonic-modulated barriers can be successfully described by a sharply-peaked barrier with a renormalized coupling constant given by (14). The renormalized coupling constant approaches the bare coupling constant $g_{1}$ when $\omega a \ll 1$. Figure 1 shows the transmission probabilities for the central band, $T_{0}=\left|t_{0}\right|^{2}$, and the first side bands, $T_{ \pm 1}$, where $T_{n}=\left|t_{n}\right|^{2}=J_{n}^{2}\left(g_{1, \mathrm{eff}}\right)$, as a function of the bare coupling constant $g_{1}$ and different values of $\omega a$. It is quite apparent that the result obtained for the sharply-peaked potential (9), corresponding to $\omega a=0$ in the plots, is a very good approximation to the square barrier even if $\omega a$ is not too small.
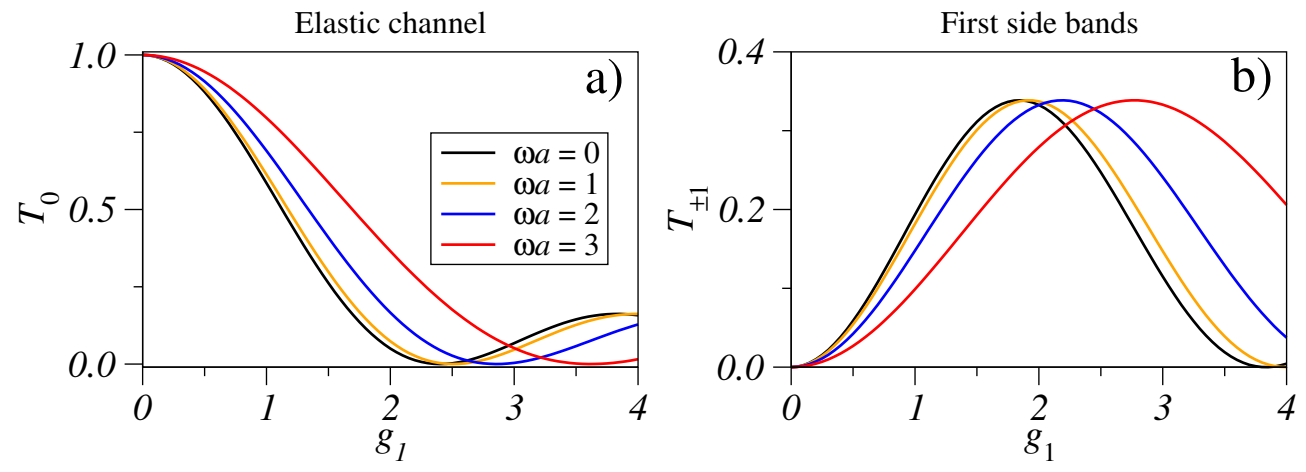

Figure 1: Transmission probabilities for a) the central band, $T_{0}$, and b) the two nearest side bands, $T_{ \pm 1}$, as a bare coupling constant $g_{1}=V_{1} a$ and different values of the parameter $\omega a$.

\section{Arbitrary barriers of finite width}

The analysis of the scattering solutions for the time-harmonic square barrier pointed out that backscattering is suppressed at the edges $x= \pm a / 2$. 
In addition, the transmission amplitudes (13) become independent of the static component of the potential, $V_{0}$. All this suggests that the transmission amplitudes for a time-harmonic barrier of arbitrary shape

$$
V(x, t)= \begin{cases}V_{0}(x)+V_{1} \cos \omega t, & -a / 2<x<a / 2 \\ 0, & \text { otherwise }\end{cases}
$$

should be exactly the same as those obtained in the previous section. The idea behind this conjecture is that any static barrier can be regarded as a superposition of narrow square barriers and heights given by $V_{0}\left(x_{i}\right), x_{i}$ being the center of each narrow barrier. But there are not multiple reflections inside the barrier region since backscattering is suppressed, then transmission should be independent of the exact shape of the static component of the potential. To validate this conjecture, we look for a solution of the form (6a). It is a matter of simple algebra to check that the wave function (6a) with the spinors

$$
\boldsymbol{A}_{n}(x)=\sum_{p=-\infty}^{\infty} A_{p} e^{i\left[E_{p} x-K_{0}(x)\right]} J_{n-p}\left(V_{1} / \omega\right) \boldsymbol{\phi}_{+}, \quad x<|a / 2|
$$

satisfies the massless Dirac equation (1) for the potential (15). Here the function $K_{0}(x)$ is defined from the relation $V_{0}(x)=d K_{0}(x) / d x$. Outside the barrier region the spinors take the form

$$
\boldsymbol{A}_{n}(x)=i^{n} \times \begin{cases}\delta_{n 0} e^{i\left[E_{n} x-K_{0}(-a / 2)\right]} \boldsymbol{\phi}_{+}, & x<-a / 2, \\ t_{n} e^{i\left[E_{n} x-K_{0}(a / 2)\right]} \boldsymbol{\phi}_{+}, & x>a / 2 .\end{cases}
$$

Finally, imposing the continuity of the wave function at $x= \pm a / 2$ and proceeding as in the previous section we arrive at (13). The conclusion from this analysis is that the static part of the potential $V(x, t)$ plays the role of a phase factor in the transmission amplitudes and consequently it does not affect the transmission probabilities $T_{n}=\left|t_{n}\right|^{2}$.

\section{Conclusions}

In summary, we have found exactly the scattering solutions of massless Dirac electrons subjected to time-harmonic barriers of arbitrary shape. The Floquet theory allows us to express the corresponding transmission amplitudes in terms of Bessel functions. In all cases the amplitudes are found to 
be independent of the incoming energy and, in the case of the sharply-peaked barrier, also of the driving frequency. Most important, we proved that the approximation of a finite-width barrier by a sharply-peaked one leads to very accurate results when $\omega a$ is not large.

This work was supported by MICINN (projects MAT2010-17180 and FIS2009-07880), JCYL (project SA226U13) and USAL (project KBBB). C. G.-S. acknowledges financial support from Comunidad de Madrid and European Social Foundation.

\section{Appendix A. Graf's addition theorem of Bessel functions}

Let us consider a triangle with sides $x, y$ and $z$. Let $\varphi$ be the angle between sides $x$ and $y$. Similarly, let $\Psi$ be the angle between sides $x$ and $z$. Graf's addition theorem of Bessel functions states that (see, e.g., page 27 of Ref. [22])

$$
J_{-m}(z) e^{-i m \Psi}=\sum_{p=-\infty}^{\infty} J_{p-m}(x) J_{p}(y) e^{i p \varphi} .
$$

We now take $x=y=V_{1} / \omega$ and $\varphi=\omega a$. From simple trigonometric considerations we get $z=\left(2 V_{1} / \omega\right)|\sin (\omega a / 2)|$ and $\Psi=(\pi-\omega a) / 2$ in this case. Therefore $\exp (-i m \Psi)=i^{-m} \exp (i m \omega a / 2)$ and (A.1) leads to equation (13).

[1] P. A. M. Dirac, Proc. R. Soc. Lond. A 117 (1928) 610.

[2] B. Thaller, The Dirac Equation, Springer-Verlag, 1992.

[3] O. Klein, Z. Phys. 53 (1929) 157.

[4] A. Calogeracos, N. Dombey, Contemp. Phys. 40 (1999) 313-321.

[5] B. H. J. McKellar, G. J. Stephenson, Phys. Rev. A 36 (1987) 2566.

[6] F. Sauter, Z. Phys. 69 (1931) 547.

[7] K. S. Novoselov, A. K. Geim, S. V. Morozov, D. Jiang, Y. Zhang, S. V. Dubonos, I. V. Grigorieva, A. A. Firsov, Science 306 (2004) 666-669.

[8] C. W. J. Beenakker, Rev. Mod. Phys. 80 (2008) 1337.

[9] P. Allain, J. Fuchs, Eur. Phys. J. B 83 (2011) 301-317. 
[10] J. M. Cerveró, E. Diez, Int. J. Theor. Phys. 50 (2011) 2134-2143.

[11] P. R. Wallace, Phys. Rev. 71 (1947) 622.

[12] M. I. Katsnelson, K. S. Novoselov, A. K. Geim, Nat. Phys. 2 (2006) 620.

[13] M. I. Katsnelson, Graphene. Carbon in two dimensions, Cambridge University Press, 2012.

[14] N. Stander, B. Huard, D. Goldhaber-Gordon, Phys. Rev. Lett. 102 (2009) 026807.

[15] A. F. Young, P. Kim, Nat. Phys. 5 (2009) 222-226.

[16] M. G. Calkin, D. Kiang, Y. Nogami, Phys. Rev. C 38 (1988) 1076-1077.

[17] F. Domínguez-Adame, J. Phys. A: Math. Gen. 23 (1990) 1993.

[18] D. F. Martinez, L. E. Reichl, Phys. Rev. B 64 (2001) 245315.

[19] B. Trauzettel, Y. M. Blanter, A. F. Morpurgo, Phys. Rev. B 75 (2007) 035305 .

[20] M. A. Zeb, K. Sabeeh, M. Tahir, Phys. Rev. B 78 (2008) 165420.

[21] W.-T. Lu, S.-J. Wang, W. Li, Y.-L. Wang, C.-Z. Ye, H. Jiang, J. Appl. Phys. 111 (2012) 103717.

[22] B. Korenev, Bessel functions and their applications, Taylor \& Francis, 2002. 